\title{
Abemaciclib Regimen
}

National Cancer Institute

\section{Source}

National Cancer Institute. Abemaciclib Regimen. NCI Thesaurus. Code C159980.

A chemotherapy regimen consisting of abemaciclib that may be used in the treatment of advanced or metastatic breast cancer. 